\title{
EAES/SAGES consensus conference on acute diverticulitis: a paradigm shift in the management of acute diverticulitis
}

\author{
Steven D. Wexner ${ }^{1} \cdot$ Mark A. Talamini $^{2}$ \\ Published online: 1 August 2019 \\ (c) Springer Science+Business Media, LLC, part of Springer Nature 2019
}

There are many differences between the 1999 and 2019 statements on diverticulitis, testifying to the evolution of the surgical treatment of this disorder. The 1999 consensus focuses on the overall diagnosis and treatment of diverticular disease, whereas the 2019 statement also includes significant information on the management of acute diverticulitis. The composition of the consensus conferences also greatly varies; the 1999 version included 16 international experts, all of whom were from Europe. Levels of evidence were not used nor was a Delphi analysis employed. In contrast, the EAES/SAGES conference that created the 2019 statement was an international project with a core group of 24 experts representing the EU, the US, and Canada. Supplementing this steering group of 24 experts and residents from the 2 societies were 2 project leads and 2 librarians. Grading of Recommendations Assessment, Development and Evaluation methodology was used. Levels of evidence were rated as high, moderate, low, or very low quality for each statement. The result is 54 consensus statements and 41 recommendations across 6 broad topic areas. The 2019 consensus document was derived from 1004 complete surveys and over 300 live votes at the diverticulitis consensus conference. The fact that consensus was achieved for 40 of 41 (97.6\%) of recommendations with 38 of $41(92 \%)$ agreement in this 2019 project is evidence of impressive success; the leaders and participants should be proud of this work. Practitioners and patients will clearly benefit from the clarity provided.

There are both similarities and differences in the 1999 and 2019 documents. In terms of similarities, both documents note that the proximal margin of resection should be soft supple bowel without the need for microscopic margin

Mark A. Talamini

mark.talamini@stonybrookmedicine.edu

1 Department of Colorectal Surgery, Cleveland Clinic Florida, Weston, FL, USA

2 Department of Surgery, Renaissance School of Medicine, Stony Brook University, Stony Brook, NY, USA assessment and/or resection of all diverticular disease. Both statements also state that primary anastomosis with loop ileostomy is preferable to a Hartmann's procedure for a variety of reasons, including initial postprocedural morbidity and the likelihood and safety of subsequent stoma reversal. The most dramatic difference is the recommended role of minimally invasive approaches in the treatment of diverticulitis. The 1999 document opines upon laparoscopy versus laparotomy, while the 2019 document addresses the nuances of laparoscopic surgery including laparoscopic lavage rather than resection, representing a significant advance during the 20-year interval. In the 1999 document, two statements stand out in this regard. "In Hinchey I and II patients, the laparoscopic approach is not the first choice, but it may be justified if no gross abnormalities are found during diagnostic laparoscopy" and "There is no place for laparoscopic resections in Hinchey III and Hinchey IV patients." We vividly recollect laparoscopic colorectal surgery between 1991 and 1998 , the first years of data collection during which opinions were formed that informed the 1999 document. We did not have the technological tools nor the technical capabilities that currently exist. Thus, these statements, in the context of 1999, were appropriate. They stand in stark contrast to the 2019 publication. As an example, question Q5.2 asks "What is the role of laparoscopic resection in emergency surgery for diverticulitis?" Answer: "Laparoscopic sigmoid resection with or without stoma in the emergency setting has been shown to decrease overall complications compared to open resections." The minimally invasive paradigm shift is also noted in the 2019 work: "When resection is indicated we recommend consideration of laparoscopic approach for perforated diverticulitis in the appropriate clinical setting." Also, question Q6.1 asks "What is the role of laparoscopy in elective surgery for diverticulitis?" Answer: "Laparoscopy is safe in the setting of elective surgery for diverticulitis and is associated with reduced rates of morbidity and length of stay compared to open surgery." Finally, "a laparoscopic approach is recommended in elective surgery for diverticular 
disease, when feasible," recommended based upon a high level of evidence and a strong strength of recommendation. The laparoscopic approach is also recommended even in the subcategories when the consensus group assessed short-term functional outcomes and quality of life, and the subcategory of obese patients.

Appropriate indications for elective resection also differ. The 1999 document proffers "patients should be considered for elective surgery if they have had at least two attacks of symptomatic diverticular disease." The current work draws a sharp distinction in opining that the majority of patients with Hinchey I or II disease who are successfully managed without surgery are unlikely to experience any further acute diverticulitis and, therefore, "surgery should not be routinely offered solely to avoid future episodes." The new document recommends a patient-specific tailored approach to elective surgical indications.

Another key issue, addressed only in the 2019 project, is the role of bowel preparation. Q6.4 asks "What is the role of bowel preparation prior to surgery in the management of diverticulitis?" Answer: "Although we found no evidence specific to diverticular disease, in a general population, the use of mechanical preparation is associated with decreased rates of SSI and anastomotic leak when compared with oral antibiotics." and "While the evidence specific to diverticular disease is limited, evidence exists in the setting of elective colorectal surgery to recommend the use of an isosmotic mechanical bowel preparation with oral antibiotics prior to surgery." This recommendation was based upon a moderate level of evidence and a strong strength of recommendation. We believe that the pendulum has swung on this topic from the traditional Nicholls-Condon combination of oral and mechanical antibiotic bowel preparation to no preparation and now back to the original recommendation of oral and mechanical bowel preparation.

The only remaining unresolved decision points in the current work are the selective use of imaging to guide diagnosis, recommendations regarding antibiotics in uncomplicated acute diverticulitis, and colonic evaluation after the resolution of uncomplicated diverticulitis.

Although there was consensus achieved upon the recommendation of selective imaging in patients with left lower quadrant pain, absence of vomiting, and CRP $>50 \mathrm{mg} / \mathrm{l}$ and/ or a prior history of acute diverticulitis, this recommendation was not felt to have been likely to change practice. In this era of cost consciousness, it makes sense that imaging is not always required particularly in some parts of the world. The second lack of consensus was that non-antibiotic therapy could be appropriate in select immuno-competent individuals. Consensus was not reached nor was this recommendation felt to change practice despite a high level of evidence with a weak grade of recommendation. This transatlantic divide may relate to fear of litigation and "failure" to conform to the "standard of care" in the US. The third area of discrepancy is routine colonic evaluation after successful treatment of uncomplicated acute diverticulitis. Although consensus was reached on the recommendation, the recommendation was not likely to change practice. Again, this difference may be due to medicolegal issues more than medical issues in the US.

We congratulate the authors of this outstanding document upon having assembled a steering committee of 28 people and administering this extensive detailed level of evidencebased and grade-based questionnaire to approximately 1300 individuals both online and in person. The differences between the documents attest to the ubiquitous acceptance of the advantages of laparoscopy realized during the 20-year interval between publications. The authors progressed from cautionary notes and contraindications to a comprehensive compendium of evidence-based guidelines about virtually every facet of the laparoscopic management of diverticulitis in both the elective and acute settings. We are confident that this EAES/SAGES consensus conference on acute diverticulitis will stand as the reference for the management of acute diverticulitis for the foreseeable future.

Publisher's Note Springer Nature remains neutral with regard to jurisdictional claims in published maps and institutional affiliations. 